Research

\title{
Cone-beam computed tomography evaluation of bone density of midpalatal suture before, after, and during retention of rapid maxillary expansion in growing patients
}

\author{
Renato Bigliazzi ${ }^{\mathrm{a}, *}$, Aline de Oliveira Silva Magalhães ${ }^{\mathrm{b}}$, Paulo Enrique Magalhães ${ }^{\mathrm{c}}$, \\ André Pinheiro de Magalhães Bertoz ${ }^{\mathrm{a}}$, Kurt Faltin Jr. ${ }^{\mathrm{b}}$, Emiko Saito Arita ${ }^{\mathrm{d}}$, \\ Francisco Antonio Bertoz ${ }^{\text {a }}$

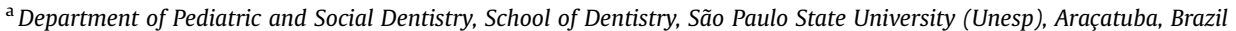 \\ ${ }^{\mathrm{b}}$ Department of Orthodontics, School of Dentistry, University Paulista, São Paulo, Brazil \\ c Private Practice in Radiology, Sorocaba, Brazil \\ ${ }^{\mathrm{d}}$ Department of Oral Radiology, School of Dentistry, University of São Paulo, São Paulo, Brazil
}

\section{A R T I C L E I N F O}

\section{Article history:}

Received 3 June 2016

Received in revised form

4 December 2016

Accepted 10 January 2017

\section{Keywords:}

Bone density

Cone-beam computed tomography

Orthopedic palatal expansion

Rapid maxillary expansion

\begin{abstract}
A B S T R A C $T$
Objective: To analyze changes in bone density of the midpalatal suture after rapid palatal expansion (RME) with the Hyrax-type expander in growing subjects using cone-beam computed tomography (CBCT).

Materials: Fourteen patients (nine girls and five boys; mean age, $11.7 \pm 2.4$ years) who required RME as part of their orthodontic treatment and had preexpansion (T0) and postexpansion (at the end of active expansion [T1], after a retention period of 3 months [T2], and after 6-month [T3] retention period) CBCT scans. On the axial CBCT images, four regions of interest in the midpalatal suture were analyzed. Bone density (BD) was measured in voxel gray-scale histogram. BD measurements were compared by using Friedman ANOVA with post hoc tests. A $P$ value less than 0.05 was considered statistically significant. Results: BD values in all regions of interest in the midpalatal suture decreased significantly after active expansion (T0-T1) and significantly increased during the retention periods (T1-T2 and T1-T3). According to the density values 3 months after expansion (T0-T2), the remineralization was incomplete. At 6 months of the retention, nonsignificant differences in BD were observed in comparison with the pretreatment values (T0-T3).

Conclusions: Active phase of RME produced effective opening of the midpalatal suture with significant decreases in BD levels. The results revealed that are necessary more than 3 months for complete sutural recovery after RME, thus indicating in prepubertal patients, the recommendation to extend the retention time to 6 months to achieve the midpalatal suture remineralization.
\end{abstract}

(c) 2017 World Federation of Orthodontists.

\section{Introduction}

Rapid maxillary expansion (RME) was reintroduced and popularized during the 1960s by Haas [1]. RME is an orthopedic procedure indicated for the treatment of maxillary transverse deficiency with or without posterior crossbite or to expand arch perimeters to alleviate dental crowding [2,3]. This effective

All authors have completed and submitted the ICMJE Form for Disclosure of Potential Conflicts of Interest, and none were reported.

* Corresponding author: Faculdade de Odontologia de Araçatuba-FOA/UNESP, Rua José Bonifácio, 1193, Araçatuba 16015-050, Brazil.

E-mail address: bigliazzir@hotmail.com (R. Bigliazzi). procedure is always able to produce transverse skeletal effects on the maxilla by opening the midpalatal suture in growing subjects regardless of the type of palatal expander [4] (bonded with acrylic coverage on the occlusal surface of posterior upper teeth or banded with the two or four anchored teeth). The midpalatal suture separates asymmetrically in a "V"-shaped pattern $[1,5,6]$, with the greatest expansion occurring in the anterior region of the palate in the horizontal plane caused by the articulation of the maxilla with the surrounding cranial bones, such as the zygomatic buttress [7]. Expansion of the midpalatal suture leads to stretching of collagenous fibers as well as new bone formation, producing a net desirable increase in the transverse width of the maxillary basal bone [8]. 
One of the major causes of postexpansion relapse is the retention period duration [9], because the velocity and the quality of bone formation at the suture might influence early post-RME relapse [10]. To minimize the relapse tendency after RME, several authors [11-16] reported that a retention period of at least 5 months is necessary; however, in a systematic review, Lione et al. [4] found that the minimum retention period to maintain the palatal expander in situ as a passive retainer was 3 months in the literature (in 17 articles of the 30 selected for the study). The use of two-dimensional (2D) images, like occlusal radiographs to evaluate a progressive ossification in the maxilla, shows only part of the midpalatal suture. The overlapping with the cranial base structures and the 2D nature of the images compromise the evaluation of the midpalatal suture ossification in its posterior area [6].

With the objective to evaluate the suture bone quality after retention post-RME using 3D computed tomography (CT), Franchi et al. [17] and Lione et al. [18], using the same sample, concluded that a retention period of 6 months was sufficient to obtain midpalatal suture bone density (BD) values similar to the pretreatment ones in growing patients. However, the use of conventional CT scans in clinical orthodontics has been limited because of cost and radiation concerns and was also supplemented by cone-beam CT (CBCT) technology with regard to the quality of the craniofacial images $[19,20]$. For these reasons, CBCT is becoming more common as a radiographic modality in orthodontic practices today $[5,21]$.

The aim of this study, therefore, was to evaluate the BD of the midpalatal suture in growing patients undergoing RME with the Hyrax-type maxillary expander using CBCT images before, at the end of active expansion, at 3 months and 6 months during the retention period.

\section{Materials}

In this retrospective study, 14 healthy white patients (mean age $11.7 \pm 2.4$ years; range, $8.5-14.8$ years; five boys, nine girls) who were treated in the Department of Orthodontics of the University Paulista and fulfilled the following inclusion criteria were selected: (1) required RME treatment because of constricted maxillary arches with or without unilateral or bilateral posterior crossbite, and prepubertal stages of cervical vertebral maturation (CS1-CS3); (2) required orthodontic treatment in the presence of unilateral or bilateral impacted canines or supernumerary teeth in the region of the upper incisors; and (3) had beginning and progress RME treatment $\mathrm{CBCT}$ scans with the ultimate goal of increased treatment efficiency or outcomes or both. All patients who fulfilled these criteria were included in the study. This study received the University Paulista Ethical Committee's approval before commencement (219.978/CEP/ICS/UNIP). The committee was aware that this was a retrospective study and it was undertaken using patient data and radiographs. All patients seeking orthodontic treatment from Faculty of Dentistry of University Paulista are informed of the possibility that all forms of their records may be use for research purposes or teaching and a written informed consent is taken with the assurance that their identity will remain anonymous.

A Hyrax palatal expander was used for each patient. The expander was activated one complete turn $(0.8 \mathrm{~mm})$ at the time it was placed, followed by two quarter turns per day $(0.4 \mathrm{~mm})$ until the expansion screw reached $8 \mathrm{~mm}$. After this process, they were stabilized. The Hyrax expander was used for retention for at least 6 months after expansion.

The i-CAT (Imaging Sciences International, Hatfield, PA) was used to obtain CBCT images before RME (TO), at the end of the active expansion phase (T1), after the retention period of 3 months (T2), and after the retention period of 6 months (T3). The first scan was taken at least 3 months before the beginning of the clinical procedures for diagnostic proposes to extend the period between the X-ray exposures. The CBCT scans were performed at $120 \mathrm{kV}$, $8 \mathrm{~mA}$, scan time of 40 seconds, $16 \times 6$ or $8-\mathrm{cm}$ field of view and $0.25-\mathrm{mm}$ voxel dimension. Raw data obtained from CBCT scanning were exported as Digital Imaging and Communications in Medicine format and imported into a specific software program (Nemotec Dental Studio 10.4; Nemotec, Madrid, Spain).

Before carrying out measurements, to minimize eventual measurement errors, the $3 \mathrm{D}$ images were oriented according to reference lines (Fig. 1). In the coronal CBCT image, reference lines were constructed passing through the center of the midpalatal suture (vertical line $U$ ) and a perpendicular line (horizontal line L) passing through the nasal cavity floor and the most superior contour of the palate. In sagittal view, the maxilla was positioned parallel to the horizontal plane (horizontal line A), passing through the area with the most contact with the maxilla, allowing visualization of the foramen perpendicular to the palatal plane and a vertical line passing through the palatine process of the maxilla. Finally, in the axial plane, the vertical line A passing through the midpalatal suture and the perpendicular line L through the palatine process of the maxilla. The point of intersection of these lines (PP) in the axial plane allows us to visualize a better symmetry between palatine halves. For the calculation of values of BD in voxel gray scale with the software radiopacity histogram, four regions of interest (ROI) in the midpalatal suture were defined (Fig. 2). In the axial image, the distance between the most anterior part of the anterior nasal spine and PP point were divided into four equal parts, determining the median points (AP, AMP, and PMP). A single trained operator made the measurements of BD values at AP, AMP, PMP, and PP ROIs and was blinded to the patient being measured.

All measurements were performed twice at 2-week intervals by the same examiner. To test the precision of the measurements in all subjects at different moments of observation, ANOVA gauge repeatability and reproducibility test was performed. This method measures the amount of variability induced in measurements by the measurement system itself, and compares it with the total variability observed to determine the viability of the measurement system. Descriptive statistics consisting of mean values and standard deviations was calculated for the density values measured. Mean differences in measurements at $\mathrm{T} 0, \mathrm{~T} 1, \mathrm{~T} 2$, and $\mathrm{T} 3$ were contrasted by means of the Friedman ANOVA for repeated measures, followed by Tukey post hoc tests at a significance level of 5\% (Table 1).

\section{Results}

The ratio of the precision of the measurement system to the (total) tolerance of the process ranged between 15.9\% and 22.0\% and was considered appropriate for the study. The four ROIs in the midpalatal suture showed a significant decrease in BD from T0 to T1, a significant increase from $\mathrm{T} 1$ to $\mathrm{T} 2$, and a lack of statistically significant differences from T0 to T3. At T2, nonsignificant differences were observed in BD at points AP and PP compared with T0, contrasting with the middle points AMP and PMP with significantly smaller density at T2. The BD values increased between $\mathrm{T} 2$ and $\mathrm{T} 3$ but significant differences were observed only at AMP and PMP ROIs. Figure 3 summarizes the means for the four ROIs at all observation periods.

\section{Discussion}

The ultimate goal of RME is to maximize skeletal effects and minimize dental effects. Furthermore, it has been the treatment chosen for correction of skeletal maxillary constriction in growing patients by many orthodontists for more than 40 years [1,22]. The 


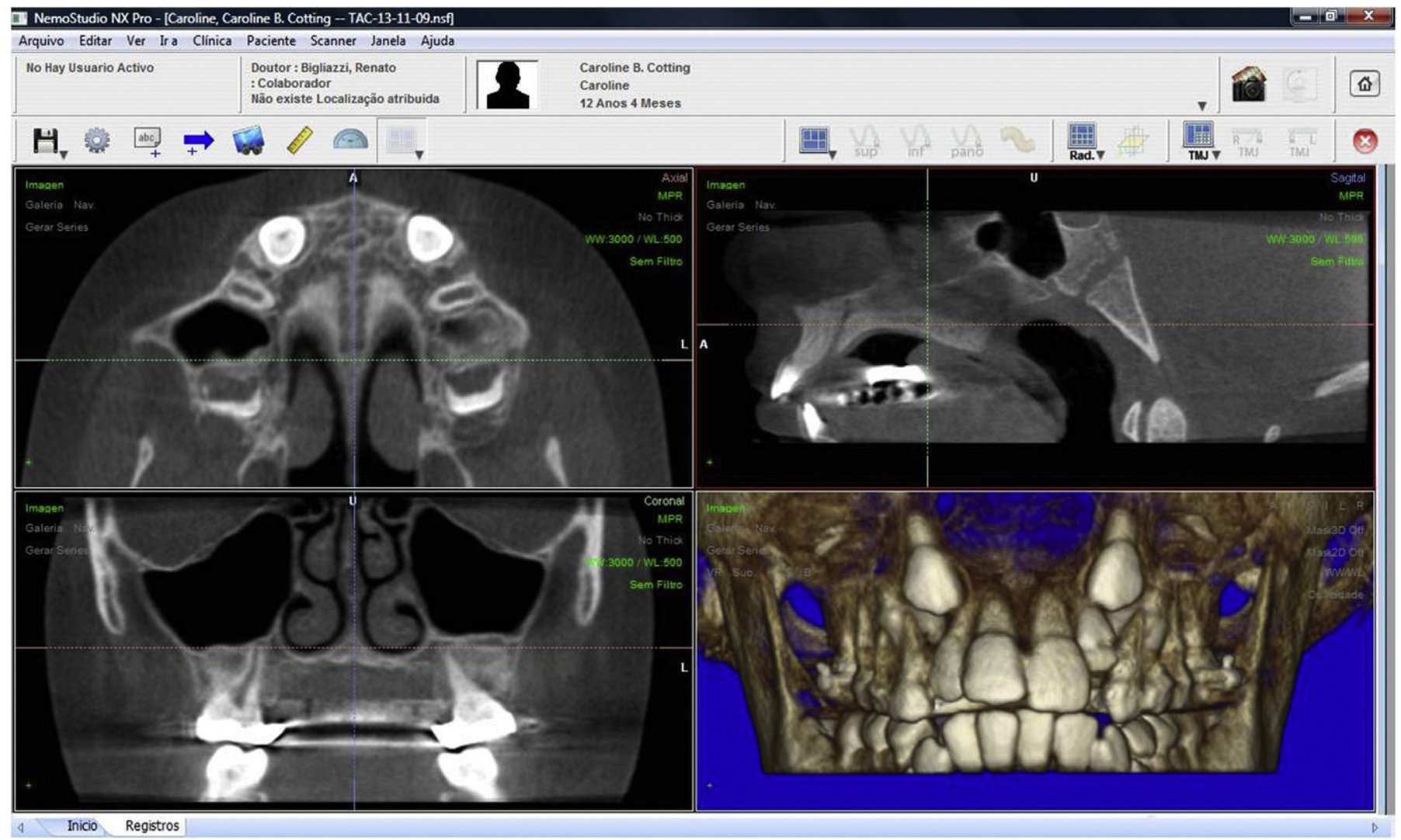

Fig. 1. Sagittal, coronal, and axial slices with the reference lines.

forces delivered by activation of RME appliance usually exceed the sutural limit and split not only the midpalatal suture but also affect other maxillary sutures [18]. The stability of the new transverse dimension is a fundamental part of the treatment, which turns the retention phase as important as the active phase [23], but the duration of the postexpansion retention period was controversial in the literature $[4,6,11-16]$. The effects of RME on the craniofacial structures and consequently the time to maintain the palatal expander device in situ to obtain stable results were generally reported based in investigations using radiological imaging [3,4,12,14-16] and histological methods [24,25]. Occlusal radiographs are not precise to assess the bone activity in the midpalatal

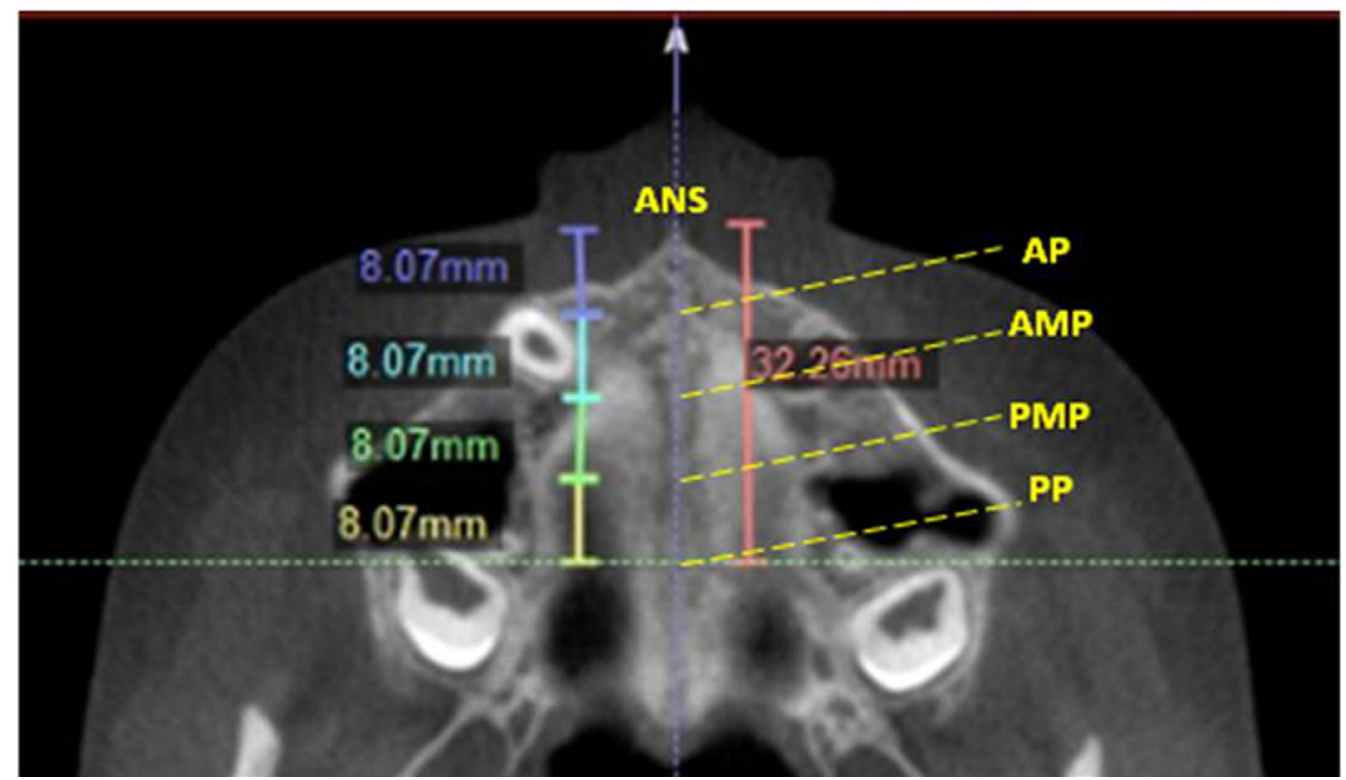

Fig. 2. Axial slice showing how the suture density measurements were recorded. 
Table 1

Descriptive statistics and statistical comparisons of density in the ROIs in the midpalatal suture at each observation period (Friedman test with Tukey post hoc tests)

\begin{tabular}{|c|c|c|c|c|c|c|c|c|c|c|}
\hline ROI & $\begin{array}{l}\text { T0 } \\
\text { Mean (SD) }\end{array}$ & $\begin{array}{l}\text { T1 } \\
\text { Mean (SD) }\end{array}$ & $\begin{array}{l}\mathrm{T} 2 \\
\text { Mean (SD) }\end{array}$ & $\begin{array}{l}\text { T3 } \\
\text { Mean (SD) }\end{array}$ & T0-T1 & T0-T2 & T0-T3 & $\mathrm{T} 1-\mathrm{T} 2$ & T1-T3 & T2-T3 \\
\hline $\mathrm{AP}$ & $382.29(261.23)$ & $25.00(113.04)$ & $254.18(229.73)$ & $357.82(255.08)$ & $0.0002^{*}$ & $0.0586 \mathrm{~ns}$ & $0.9588 \mathrm{~ns}$ & $0.0003^{*}$ & $0.0002^{*}$ & $0.1661 \mathrm{~ns}$ \\
\hline AMP & 444.68 (191.95) & $78.68(101.43)$ & $290.57(182.03)$ & $384.43(162.70)$ & $0.0002^{*}$ & $0.0003^{*}$ & $0.2652 \mathrm{~ns}$ & $0.0002^{*}$ & $0.0002^{*}$ & $0.0310^{*}$ \\
\hline PMP & $372.82(174.58)$ & $30.04(108.21)$ & 239.43 (177.49) & $368.71(161.80)$ & $0.0002^{*}$ & $0.0077^{*}$ & $0.9996 \mathrm{~ns}$ & $0.0002^{*}$ & $0.0002^{*}$ & $0.0102^{*}$ \\
\hline PP & $387.04(185.92)$ & $73.00(89.63)$ & $316.29(123.60)$ & $390.32(115.34)$ & $0.0002^{*}$ & $0.3292 \mathrm{~ns}$ & $0.9998 \mathrm{~ns}$ & $0.0002^{*}$ & $0.0002^{*}$ & $0.2906 \mathrm{~ns}$ \\
\hline
\end{tabular}

ns, nonsignificant; ROI, region of interest; SD, standard deviation.

* Statistically significant $(P<0.05)$.

suture and its surrounding tissues. Histological findings, on the other hand, are limited to experimental studies [26,27].

Recently, to overcome the limitations of conventional 2D radiographic images, studies using $\mathrm{CBCT}$ to evaluate the skeletal and dental effects of RME have become more abundant. CBCT enables a 3D visualization of the whole craniofacial complex, with precise and reliable measurements of the changes caused by RME $[2,5]$. The relatively low radiation doses with high spatial resolution and accurate $3 \mathrm{D}$ views allow thorough information to be obtained for the bone dimensions and quality (e.g., the BD) $[28,29]$. The ever-improving image quality of CBCT allows it to display trabecular bone patterns, indicating that it may be possible to apply structural analysis methods that are commonly used in micro-CT and histology [30].

Although long-term skeletal changes have been examined $[3,31,32]$, there is still no clear statement about the minimal retention time in growing patients after RME. Usually the retention period varies between 3 and 6 months [17,18,33]. The present study evaluates the midpalatal suture's BD in growing patients, before, after active RME, and after 3 and 6 months of retention. After active expansion ( $8 \mathrm{~mm}$ of expansion at the level of the screw), a significant reduction of $\mathrm{BD}$ in all ROIs studied in the midpalatal suture (T0-T1) was observed. This confirms the orthopedic opening in all suture extensions observed in previous studies [2,5-7,17,18]. After the 3-month retention period, a significant increase in BD was observed (T1-T2), but this increase was not sufficient for total mineral bone reorganization of the suture. In the middle portion of the suture, significantly low density values at the AMP and PMP ROIs were observed in comparison with density values before RME (T0-T2). After the 6-month retention period in all subjects, the BD levels increased further (T2-T3); the midpalatal suture appeared reorganized with density values similar to the pretreatment values (T0-T3). This finding is consistent with previous observations in which a low-dose CT image was used [17,18], thus indicating further that a retention period longer than 3 months may be appropriate for recovering the bone mineral density of the midpalatal suture, reducing the amount of skeletal relapse $[3,6,15]$. Skeletal relapse may be a result of a reactive force, called the buttressing effect, which develops against the expansion forces according to patient age, and the circummaxillary bone rigidity [34]. The present study revealed a higher increase in $\mathrm{BD}$ at the anterior (AP ROI) and posterior regions (PP ROI) compared with the middle portion of the suture (AMP and PMP ROIs) during the retention period, not confirming the posteroanterior closing pattern (like a "zip") of the midpalatal suture [25].

In conclusion, clinical patient $\mathrm{CBCT}$ images can detect the relative difference in $\mathrm{BD}$ distribution in the midpalatal suture before and after RME by using the same CBCT scanner, opening the possibility in future studies to determine if the amount and velocity of bone remineralization in growing patients are correlated with the amount of opening of the midpalatal suture during RME. Additional studies are needed to determine precisely the minimum amount of BD in the midpalatal suture in growing patients as a predictor to avoid transverse relapse after RME. Utilization of CBCT

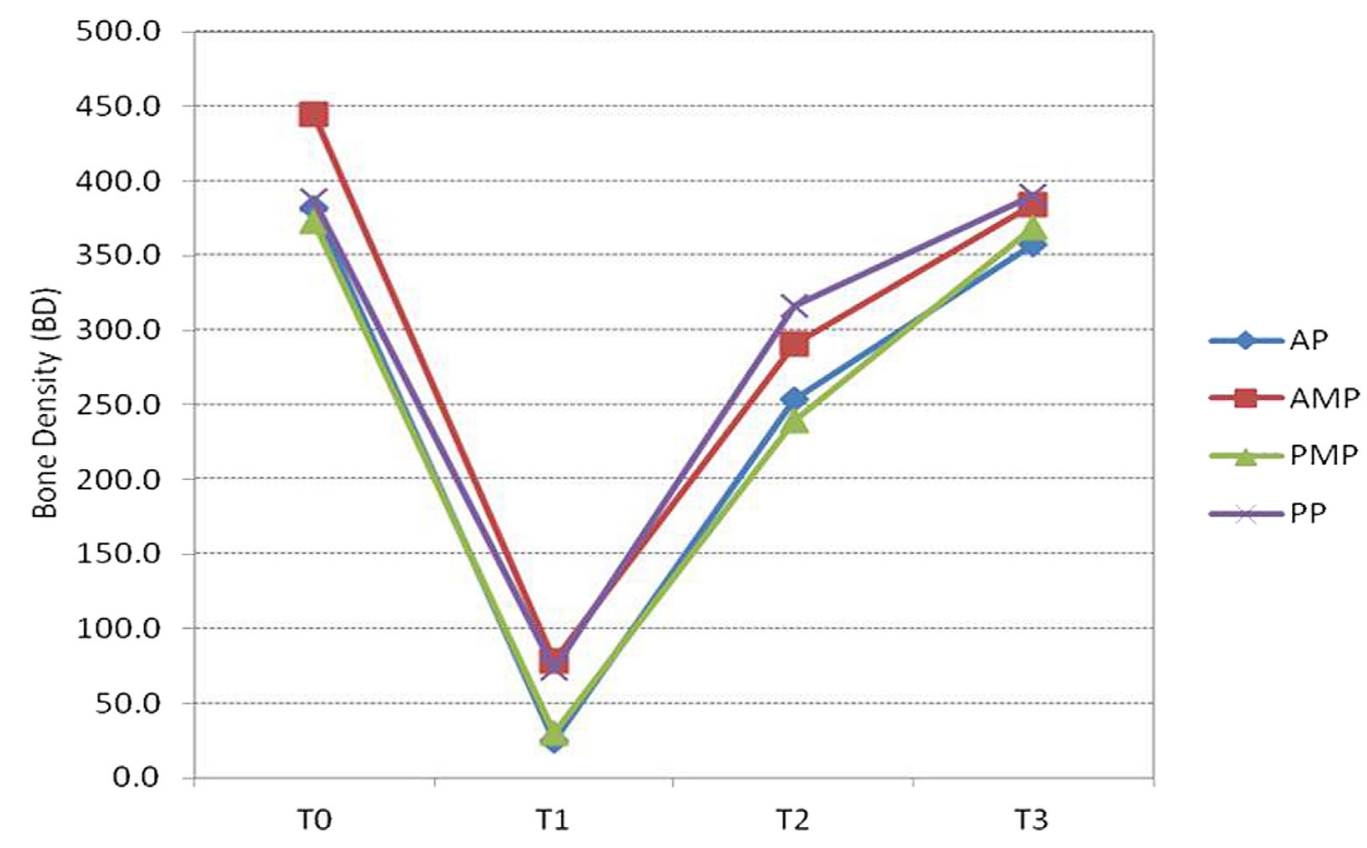

Fig. 3. Means of BD values for the four ROIs in all observation periods. 
images in subjects undergoing RME might allow better control in the retention period and could help orthodontists in the daily routine.

\section{Conclusions}

- The active phase of RME produced significant decreases in BD levels, thus indicating effective opening of the midpalatal suture.

- According to the density values 3 months after RME, the remineralization of the midpalatal suture was not complete.

- Our results support the recommendation to extend RME retention time to 6 months to prevent transverse relapse.

- The CBCT is a reliable method for assessing changes in the BD in the midpalatal suture following RME.

\section{References}

[1] Haas AJ. Rapid expansion of the maxillary dental arch and nasal cavity by opening the midpalatal suture. Angle Orthod 1961;31:73-89.

[2] Garrett BJ, Caruso JM, Rungcharassaeng K, Farrage JR, Kim JS, Taylor GD. Skeletal effects to the maxilla after rapid maxillary expansion assessed with cone-beam computed tomography. Am J Orthod Dentofacial Orthop 2008;134:8-9.

[3] Lagravere MO, Major PW, Flores-Mir C. Long-term skeletal changes with rapid maxillary expansion: a systematic review. Angle Orthod 2005;75:1046-52.

[4] Lione R, Franchi L, Cozza P. Does rapid maxillary expansion induce adverse effects in growing subjects? Angle Orthod 2013;83:172-82.

[5] Weissheimer A, de Menezes LM, Mezomo M, Dias DM, Santayana de Lima EM, Deon Rizzatto SM. Immediate effects of rapid maxillary expansion with Haastype and hyrax-type expanders: a randomized clinical trial. Am J Orthod Dentofacial Orthop 2011;140:366-76.

[6] da Silva Filho OG, Lara TS, da Silva HC, Bertoz FA. Post expansion evaluation of the midpalatal suture in children submitted to rapid palatal expansion: a CT study. J Clin Pediatr Dent 2006;31:142-8.

[7] Marcotte MR. The instantaneous transverse changes in the maxilla due to different points of force application. J Dent Res 1977;56:465-70.

[8] McNamara JA, Brudon WL. Orthodontics and dentofacial orthopedics. Ann Arbor, MI: Needham Press; 1995. p. 211-2.

[9] Iseri H, Ozsoy S. Semirapid maxillary expansion-a study of long-term transverse effects in older adolescents and adults. Angle Orthod 2004; $74: 71-8$.

[10] Saito S, Shimizu N. Stimulatory effects of low-power laser irradiation on bone regeneration in midpalatal suture during expansion in the rat. Am J Orthod Dentofacial Orthop 1997;111:525-32.

[11] Isaacson RJ, Murphy TD. Some effects of rapid maxillary expansion in cleft lip and palate patients. Angle Orthod 1964;34:143-54.

[12] Haas AJ. Palatal expansion: just the beginning of dentofacial orthopedics. Am J Orthod 1970;57:219-55.

[13] Ekstrom C, Henrickson CO, Jensen R. Mineralization in the midpalatal suture after orthodontic expansion. Am J Orthod 1977;71:449-55.

[14] Bell RA. A review of maxillary expansion in relation to rate of expansion and patient's age. Am J Orthod 1982;81:32-7.
[15] Mew J. Relapse following maxillary expansion: a study of twenty-five consecutive cases. Am J Orthod Dentofacial Orthop 1983;83:56-61.

[16] Bishara SE, Taley RN. Maxillary expansion: clinical implications. Am J Orthod Dentofacial Orthop 1987;91:3-14.

[17] Franchi L, Baccetti T, Lione R, Fanucci E, Cozza P. Modifications of midpalatal sutural density induced by rapid maxillary expansion: a low-dose computed-tomography evaluation. Am J Orthod Dentofacial Orthop 2010;137:486-8.

[18] Lione R, Franchi L, Fanucci E, Laganà G, Cozza P. Three-dimensional densitometric analysis of maxillary sutural changes induced by rapid maxillary expansion. Dentomaxillofac Radiol 2013;42:71798010.

[19] De Vos W, Casselman J, Swennen GR. Cone-beam computerized tomography $(\mathrm{CBCT})$ imaging of the oral and maxillofacial region: a systematic review of the literature. Int J Oral Maxillofac Surg 2009;38:609-25.

[20] Cassetta M, Stefanelli LV, Di Carlo S, Pompa G, Barbato E. The accuracy of $\mathrm{CBCT}$ in measuring jaws bone density. Eur Rev Med Pharmacol Sci 2012;16:1425-9.

[21] van Vlijmen OJ, Kuijpers MA, Bergé SJ, et al. Evidence supporting the use of cone-beam computed tomography in orthodontics. J Am Dent Assoc 2012;143:241-52.

[22] Isaacson RJ, Ingram AH. Forces produced by rapid maxillary expansion. Part II: forces present during treatment. Angle Orthod 1964;34:261-70.

[23] Lima Filho RM, de Oliveira Ruellas AC. Long-term maxillary changes in patients with skeletal Class II malocclusion treated with slow and rapid palatal expansion. Am J Orthod Dentofacial Orthop 2008;134:383-8.

[24] Cleall JF, Bayne DI, Posen JM, Subtelny JD. Expansion of the midpalatal suture in the monkey. Angle Orthod 1965;35:23-35.

[25] Vardimon AD, Brosh T, Spiegler A, Lieberman M, Pitaru S. Rapid palatal expansion: part 1. Mineralization pattern of the midpalatal suture in cats. Am J Orthod Dentofacial Orthop 1998;113:371-8.

[26] Baydas B, Yavuz I, Uslu H, Dagsuyu IM, Ceylan I. Nonsurgical rapid maxillary expansion effects on craniofacial structures in young adult females. A bone scintigraphy study. Angle Orthod 2006;76:759-67.

[27] Arat ZM, Gökalp H, Atasever T, Türkkahraman H. ${ }^{99 m}$ Technetium-labeled methylene diphosphonate uptake in maxillary bone during and after rapid maxillary expansion. Angle Orthod 2003;73:545-9.

[28] Metzler P, Zemann W, Lübbers HT, et al. Bone mineral density measurements performed by cone-beam computed tomography in the bisphosphonaterelated osteonecrosis-affected jaw. Oral Radiol 2012;28:101-8.

[29] Han S, Bayome M, Lee J, Lee YJ, Song HH, Kook YA. Evaluation of palatal bone density in adults and adolescents for application of skeletal anchorage devices. Angle Orthod 2012;82:625-31.

[30] Pauwels R, Jacobs R, Singer SR, Mupparapu M. CBCT-based bone quality assessment: are Hounsfield units applicable? Dentomaxillofac Radiol 2015;44:61-76.

[31] Ferris T, Alexander RG, Boley J, Buschang PH. Long-term stability of combined rapid palatal expansion-lip bumper therapy followed by full fixed appliances. Am J Orthod Dentofacial Orthop 2005;128:310-25.

[32] Bucci R, D’Antò V, Rongo R, Valletta R, Martina R, Michelotti A. Dental and skeletal effects of palatal expansion techniques: a systematic review of the current evidence from systematic reviews and meta-analyses. J Oral Rehabil 2016;43:543-64.

[33] Korbmacher H, Huck L, Merkle T, Kahl-Nieke B. Clinical profile of rapid maxillary expansion-outcome of a national inquiry. J Orofac Orthop 2005;66:455-68.

[34] Acar YB, Motro M, Erverdi AN. Hounsfield Units: a new indicator showing maxillary resistance in rapid maxillary expansion cases? Angle Orthod 2015;85:109-16. 